\title{
Enhanced Epithelial Gene Transfer by Modulation of Tight Junctions with Sodium Caprate
}

\author{
Carolyn B. Coyne, Miriam M. Kelly, Richard C. Boucher, and Larry G. Johnson \\ Departments of Pharmacology and Medicine, and Cystic Fibrosis/Pulmonary Research and Treatment Center, University of North \\ Carolina at Chapel Hill, Chapel Hill, North Carolina
}

\begin{abstract}
The airway epithelium is resistant to infection by gene transfer vectors when infected from the luminal surface. One strategy for enhancing airway epithelial gene transfer is to modify paracellular permeability, thereby permitting the diffusion of vectors to the basolateral surface, where uptake receptors are expressed. We investigated the ability of a medium-chain fatty acid known to enhance drug absorption, sodium caprate (C10), to increase airway paracellular permeability in comparison with ethyleneglycol-bis-( $\beta$-aminoethyl ether)- $N, N^{\prime}$-tetraacetic acid (EGTA). Apical application of C10 decreased transepithelial resistance by $>90 \%$ within minutes, whereas EGTA required an hour or more to produce a similar effect. C10 increased mannitol and dextran permeability by sevenfold, as compared with a twofold increase produced by EGTA. A greater enhancement of adenoviral lac $Z$ gene transfer was mediated by $\mathrm{C} 10$ ( 50 -fold over controls) than by EGTA (10fold over controls). This correlated with a significant enhancement of adenoviral CFTR-mediated correction of $\mathrm{Cl}^{-}$transport in polarized human airway epithelial (HAE) cells from cystic fibrosis (CF) patients. Confocal microscopy revealed a redistribution of claudin-1 following C10 but not EGTA treatment as a possible mechanism of gene-transfer enhancement by $\mathrm{C} 10$. These data suggest that C10 may be a better agent for enhancing gene transfer than is EGTA, and that this effect occurs through disruption of claudin-1.
\end{abstract}

Gene transfer to airway epithelium for the treatment of cystic fibrosis (CF) is an attractive therapeutic strategy. However, airway epithelia are resistant to infection by adenoviral (Ad), adeno-associated viral (AAV), retroviral, and liposomal vectors when transduced from the luminal surface, limiting the potential effectiveness of this therapy (1-4). In contrast, transduction of airway epithelial cells from the basolateral surface yields higher levels of overall gene transfer. The polarity of infection by Ad, AAV, and retroviral vectors for airway epithelia is a direct result of the asymmetric distribution of viral receptors. Ad infection requires the initial binding of the viral fiber knob proteins to the Coxsackie virus $\mathrm{B}$ and $\mathrm{Ad} 2 / 5$ receptor, followed by internalization of the virus mediated by $\alpha_{v} \beta_{3 / 5}$ integrins. Both CAR and $\alpha_{v} \beta_{3 / 5}$ integrins are localized predominantly on the basolateral surface of the airway epithelium $(5,6)$. This basolateral localization of viral recep-

(Received in original form March 9, 2000 and in revised form June 26, 2000)

Address correspondence to: Larry G. Johnson, M.D., Associate Professor of Medicine, CF/Pulmonary Research and Treatment Center, 7123A Thurston Bowles Building, CB\# 7248, UNC-CH, Chapel Hill, NC 275997248. E-mail: gdoc@med.unc.edu

Abbreviations: adenoviral, Ad; adenoviral cystic fibrosis transmembrane regulatory gene, AdCFTR; Dulbecco's modified Eagle's medium, DMEM; ethylene-glycol-bis-( $\beta$-aminoethyl ether)- $N, N^{\prime}$-tetraacetic acid, EGTA; human airway epithelial, HAE; multiplicity of infection, MOI; sodium caprate, $\mathrm{C} 10$; transepithelial resistance, $\mathrm{R}_{\mathrm{t}}$; tight junction, TJ; Zonula occludens, ZO.

Am. J. Respir. Cell Mol. Biol. Vol. 23, pp. 602-609, 2000

Internet address: www.atsjournals.org tors is also found with the AAV heparan sulfate receptor, and functional evidence suggests that receptors for amphotropic and pseudotyped retroviral vectors, such as the vesicular stomatitis virus glycoprotein $(\mathrm{G})$ receptor, may also be basolaterally localized $(1,7)$.

One strategy for increasing gene transfer to airway epithelium involves the delivery of viral vectors to the basolateral surface, to allow for viral binding and internalization. However, a characteristic of airway epithelium is its ability to prevent the penetration of foreign antigens into the interstitial space. The intercellular junctional complex of airway epithelia, composed of the tight junction (TJ) and adherens junction, serves as a barrier to this penetration.

Numerous agents have been identified that disrupt the integrity of the intercellular junctional complex. Extracellular $\mathrm{Ca}^{2+}$ chelators, such as ethyleneglycol-bis-( $\beta$-aminoethyl ether)- $N, N^{\prime}$-tetraacetic acid (EGTA), can reversibly increase the permeability of the TJ, and have been shown to enhance airway epithelial gene transfer $(1,3,8)$. However, the effect of EGTA on the integrity of proteins localizing to the airway epithelial TJ is relatively unknown. Sodium caprate $(\mathrm{C} 10)$, the sodium salt of the saturated medium-chain fatty acid capric acid, has also been shown to increase the permeability of the TJ, and has been used as an enhancer for drug absorption in products marketed in Japan, Denmark, and Sweden (9). Although the effects of this agent on tight-junctional proteins on intestinal cells have been studied, its effects on the airway epithelium are unknown.

We compared the effects of $\mathrm{C} 10$ on the transepithelial resistance $\left(\mathrm{R}_{\mathrm{t}}\right)$ and paracellular permeability $\left(\mathrm{P}_{\mathrm{app}}\right)$ of human airway epithelial (HAE) cells with the effects of EGTA, the agent most typically used to enhance airway gene transfer $(1,3,8)$. We then investigated whether regulation of $\mathrm{TJ}$ permeability by $\mathrm{C} 10$ would allow the penetration of viral vectors to the basolateral surfaces of HAE cells. Subsequently, we attempted to correlate increased paracellular permeability with changes in gene transfer efficiency. To address potential toxicity issues in vitro, we examined the effects of C10 and EGTA on cytokine production by HAE cells. To correlate enhancement of gene transfer with the localization of airway tight-junctional proteins, we evaluated the effects of C10 and EGTA on protein components of the TJ by immunofluorescence localization and Western blot analysis of Zonula occludens (ZO)-1, occludin, E-cadherin, and claudin-1.

\section{Materials and Methods Cell Culture}

Transformed cells. NIH 3 T3 cells were cultured on plastic dishes at $37^{\circ} \mathrm{C}$ (in a humidified atmosphere of $5 \% \mathrm{CO}_{2} /$ air) with Dul- 
becco's modified Eagle's medium (DMEM) containing glucose at $4.5 \mathrm{~g} / \mathrm{ml}$ (DMEM-high glucose [H]), 5\% fetal bovine serum, and $500 \mu \mathrm{g} / \mathrm{ml}$ penicillin/streptomycin. After trypsin digestion, cells were suspended in DMEM-H and plated at a density of $10^{5}$ cells per $12 \mathrm{~mm}(0.4-\mu \mathrm{m}$ pore size) Transwell-Col inserts (Costar, Cambridge, MA).

Polarized Primary HAE Cells. Nasal and bronchial cells of normal and CF type were isolated from surgical specimens (10), plated at a density of $10^{5}$ cells $/ 12 \mathrm{~mm}(0.4-\mu \mathrm{m}$ pore size) Transwell-Col insert, and maintained in a 50:50 mixture of LHC Basal Medium (Biofluids, Rockville, MD) and DMEM-H medium supplemented with growth factors, retinoic acid, and bovine serum albumin (BSA). After cultures reached confluence at $\sim 7 \mathrm{~d}$, medium was aspirated from the apical surface and cells were maintained at an air-liquid interface for 2 to $3 \mathrm{wk}$. Cells with more than $10 \%$ cilia as determined by microscopy, and an $R_{t}>600$ $\Omega-\mathrm{cm}^{2}$, were used for experiments.

\section{Measurement of Transepithelial Resistance $\left(R_{t}\right)$}

The $\mathrm{R}_{\mathrm{t}}$ of primary HAE cells was monitored with an ohmmeter (EVOM; World Precision Instruments, Sarasota, FL). Medium was added to the apical surface and basolateral compartments, and $\mathrm{R}_{\mathrm{t}}$ was measured between electrodes.

\section{Transepithelial Cell Permeability}

Permeation of $\left[{ }^{3} \mathrm{H}\right]$ mannitol (MW: $182 \mathrm{kD}$; Sigma, St. Louis, MO) and $\left[{ }^{14} \mathrm{C}\right]$ dextran (MW: 2,000 kD; Sigma) across primary HAE cells was measured after treatment with 10 mM EGTA, 30 $\mathrm{mM} \mathrm{C} 10$, or medium alone. $\left[{ }^{3} \mathrm{H}\right]$ Mannitol $(5 \mu \mathrm{Ci})$ and $\left[{ }^{14} \mathrm{C}\right] \mathrm{dex}-$ tran $(5 \mu \mathrm{Ci})$ were added to the apical compartment, and samples $(10 \mu \mathrm{l})$ were removed from the apical and basolateral compartments at 10, 20,30, 40, and 60 min. Radioactivity in each sample was counted, and the $\mathrm{P}_{\text {app }}$ coefficient was calculated (11) directly after treatment and again at $12 \mathrm{~h}$ and $24 \mathrm{~h}$ after treatment.

\section{Recombinant Adenoviruses}

Recombinant, first-generation E1,E3-deleted adenovirus serotype 5 vectors containing Escherichia coli lac Z (AdlacZ) or CF transmembrane conductance regulator (CFTR) (AdCFTR) complementary DNA (cDNA) were prepared by the University of North Carolina at Chapel Hill Gene Therapy Center Vector Core. The vector titers were approximately $10^{11}$ transducing units $/ \mathrm{ml}$ for each vector.

\section{Ad Infections}

Cultures of primary HAE cells were treated with vehicle, $10 \mathrm{mM}$ EGTA, or $30 \mathrm{mM} \mathrm{C10}$. When $\mathrm{R}_{\mathrm{t}}$ values of C10- or EGTA-treated cultures had decreased to $<90 \%$ of their initial levels (at 2 to 5 min for $\mathrm{C} 10$ and $\sim 60$ min for EGTA), cells were washed with phosphate-buffered saline (PBS) and infected with AdlacZ at a multiplicity of infection (MOI) of 100 or with AdCFTR at an MOI of 300. After infection for $2 \mathrm{~h}$ at $37^{\circ} \mathrm{C}$, cells were washed with PBS and incubated for an additional $48 \mathrm{~h}$. Transduction efficiency was measured with the Galactostar Light assay (Tropix, Bedford, MA).

\section{Labeling of Adenovirus}

For labeling of Ad vectors, carbocyanine dye (Cy3) (Amersham) was covalently linked to their capsid proteins as previously described (12).

\section{Immunofluorescence Labeling and Confocal Microscopy}

Cells were permeabilized with methanol at $-20^{\circ} \mathrm{C}$ for $30 \mathrm{~min}$. Rabbit polyclonal antibodies to human ZO-1, occludin, or claudin-1 (Zymed Laboratories, San Francisco, CA), diluted 1:1,000, or mouse monoclonal antihuman E-cadherin antibody (Zymed) diluted 1:2,000 in PBS/BSA $(1 \mathrm{mg} / \mathrm{ml})$, were added to the luminal surface of the treated cells for $1 \mathrm{~h}$. Cells were washed with PBS, and Texas Red-labeled antirabbit or antimouse secondary antibody (Amersham), diluted 1:600 in 10\% goat serum/PBS, was added to the luminal surface. Cells were then postfixed with $4 \%$ paraformaldehyde. Transwell-Col inserts were excised from the plastic holder and mounted on slides with $100 \mu$ l Vectashield (Vector Laboratories, Burlingame, CA) containing 4',6-diamidino2-phenylindole. Images were captured with a confocal laser-scanning microscope (Leica, Exton, PA), with the same settings used for images of control, EGTA-treated, and C10-treated cultures. To analyze staining intensity, we compared the intensity of immunofluorescence in multiple fields of cultures following EGTA or C10 treatment $(n=3)$ with the intensity in control cultures, using Metamorph image analysis (Universal Imaging, West Chester, PA).

\section{Western Blotting}

Lysates of primary HAE cells were prepared with $100 \mu$ l of urea buffer (13) or $0.1 \%$ Triton X-100 extraction buffer. Equal amounts of protein $(30 \mu \mathrm{g})$ were loaded onto Tris-glycine gels (Novex, San Diego, CA). After electrophoresis, protein was transferred to nitrocellulose membranes and blocked in 5\% fat-free milk, and the membranes were probed with antihuman ZO-1 $(1: 2,000)$, occludin $(1: 1,000)$, E-cadherin $(1: 1,500)$, CFTR (1:600), or claudin-1 $(1: 1,000)$ antibodies in Tris-buffered saline-Tween-20 (TBS-T). Protein was visualized with a peroxidase-conjugated secondary antibody $(1: 20,000)$ by enhanced chemiluminescence (ECL). Protein bands were located with an ECL system (Amersham).

\section{Bioelectric Characterization of CFTR-mediated $\mathrm{Cl}^{-}$Transport}

Cultured CF HAE cells grown on 12-mm Transwell-Col inserts were mounted in modified Ussing chambers interfaced with an electrometer, in which transepithelial potential difference $\left(V_{t}\right)$ and resistance were monitored continuously (13). Basal $V_{t}$, resistance, and current were recorded, and the sequential effects of amiloride $\left(10^{-4} \mathrm{M}\right)$, luminal $\mathrm{Cl}^{-}$substitution, forskolin $\left(10^{-5} \mathrm{M}\right)$, and uridine triphosphate $\left(10^{-5} \mathrm{M}\right)$ on these parameters in AdCFTRinfected and control cultures were measured.

\section{Cytokine Assays}

Normal and CF HAE cells grown on Transwell-Col inserts were treated with vehicle, $10 \mathrm{mM}$ EGTA, or $30 \mathrm{mM} \mathrm{C10}$. As a positive control, $5 \mu \mathrm{g}$ lipopolysaccharide (LPS; Sigma) was added bilaterally to the medium of parallel cultures for $1 \mathrm{~h}$. Interleukin (IL)-6 and IL- 8 secretion into the medium of all cultures was measured at $4 \mathrm{~h}$ after treatment with an enzyme-linked immunosorbent assay (Duoset, R\&D Systems, Minneapolis, MN).

\section{Statistics}

Data are presented as mean \pm SEM. One-way analysis of variance and Dunnett's correction for multiple comparisons were used to determine statistical significance (13).

\section{Results}

\section{Effects of Tight-Junctional Regulating Agents on Permeability}

To identify agents that modulated the permeability of the TJ in primary HAE cells, we examined the effects on $R_{t}$ of agents chosen from the literature. Agents that reduced $R_{t}$ rapidly $(<2 \mathrm{~h})$ and allowed for full recovery of $R_{t}$ were selected as candidates. Of the agents screened, EGTA and 
TABLE 1

Effect of tight-junctional agents on transepithelial resistance*

\begin{tabular}{lccc}
\hline $\begin{array}{l}\text { Tight-Junctional } \\
\text { Agent }\end{array}$ & $\begin{array}{c}\text { Time to Decrease in } \mathrm{R}_{\mathrm{t}} \\
\text { 16HBE14o or MDCK }\end{array}$ & $\begin{array}{c}\text { Time to Decrease in } \mathrm{R}_{\mathrm{t}} \\
\text { Primary HAE cells }\end{array}$ & Reversible \\
\hline Anti-E-cadherin antibody & $24 \mathrm{~h}$ & Infinite & NA \\
Cytochalasin D & $2 \mathrm{~h}$ & $6 \mathrm{~h}$ & No \\
Genistein & Infinite & Infinite & NA \\
Phenylarsine oxide & $6 \mathrm{~h}$ & $6 \mathrm{~h}$ & Yes \\
Polidocanol & Immediate & Immediate & No \\
EGTA & $2 \mathrm{~h}$ & $1-2 \mathrm{~h}$ & Yes \\
Sodium caprate $(\mathrm{C} 10)$ & $2 \mathrm{~min}$ & $2 \mathrm{~min}$ & Yes \\
\hline
\end{tabular}

Definition of abbreviations: EGTA = ethyleneglycol-bis-( $\beta$-aminoethyl ether)- $N, N^{\prime}$-tetraacetic acid; HAE = human airway epithelial; MDCK = Madin-Darby canine kidney cells; $\mathrm{NA}=$ not applicable; $\mathrm{R}_{\mathrm{t}}=$ transepithelial resistance; $16 \mathrm{HBEO}^{-}=$human airway cell line.

*Agents included an antibody, detergent, cytoskeletal agent, tyrosine phosphatase inhibitor, calcium chelator, and medium-chain fatty acid.

C10 met these criteria best (Table 1). The optimal concentrations of these agents were $10 \mathrm{mM}$ EGTA and $30 \mathrm{mM}$ C10. The kinetics of these two agents are shown in Figure 1A. Whereas EGTA reduced $R_{t}$ to levels similar to those following treatment with $\mathrm{C} 10$, this decrease in $\mathrm{R}_{\mathrm{t}}$ required a $1 \mathrm{~h}$ incubation. In contrast, treatment with $\mathrm{C} 10$ decreased $\mathrm{R}_{\mathrm{t}}$ to $5 \%$ of initial levels within 5 min of treatment. By $24 \mathrm{~h}$ after treatment, $\mathrm{R}_{\mathrm{t}}$ of $\mathrm{C} 10$-treated cultures returned to $108 \%$ of control levels, whereas $R_{t}$ of EGTA-treated cultures had recovered to $83 \%$ of control levels. Thus, we determined that $\mathrm{C} 10$ decreased $\mathrm{R}_{\mathrm{t}}$ more quickly than did EGTA, and allowed for full recovery by $24 \mathrm{~h}$ after treatment.

To correlate changes in $\mathrm{R}_{\mathrm{t}}$ with changes in paracellular permeability, we performed radioisotopic flux analyses of nonpolar solutes. The $\mathrm{P}_{\mathrm{app}}$ of a small molecule, mannitol
$(\mathrm{MW}=182 \mathrm{kD})$, and that of a large molecule whose molecular weight approximates that of an adenovirus, dextran $(\mathrm{MW}=2,000 \mathrm{kD})$, were measured over time in parallel vehicle-, EGTA-, or C10-treated cultures. Cultures treated with either $10 \mathrm{mM}$ EGTA or $30 \mathrm{mM} \mathrm{C10}$ displayed a much higher $\mathrm{P}_{\mathrm{app}}$ with both $\left[{ }^{3} \mathrm{H}\right]$ mannitol and $\left[{ }^{14} \mathrm{C}\right]$ dextran than did cultures treated with vehicle alone (Figure 1B). However, by $24 \mathrm{~h}$ after treatment, $\mathrm{P}_{\text {app }}$ had returned to levels similar to those in cultures treated with vehicle alone (Figure $1 \mathrm{C}$ ). These data suggest that as the $\mathrm{R}_{\mathrm{t}}$ of these monolayer cultures recovered, the permeability of both large and small molecules returned to baseline levels. Although both EGTA and C10 reduced $\mathrm{R}_{\mathrm{t}}$ to similar levels (Figure 1A), our data demonstrate that $\mathrm{C} 10$ caused a significant increase in $\mathrm{P}_{\mathrm{app}}$ over that in cultures treated with EGTA (Figure 1B).
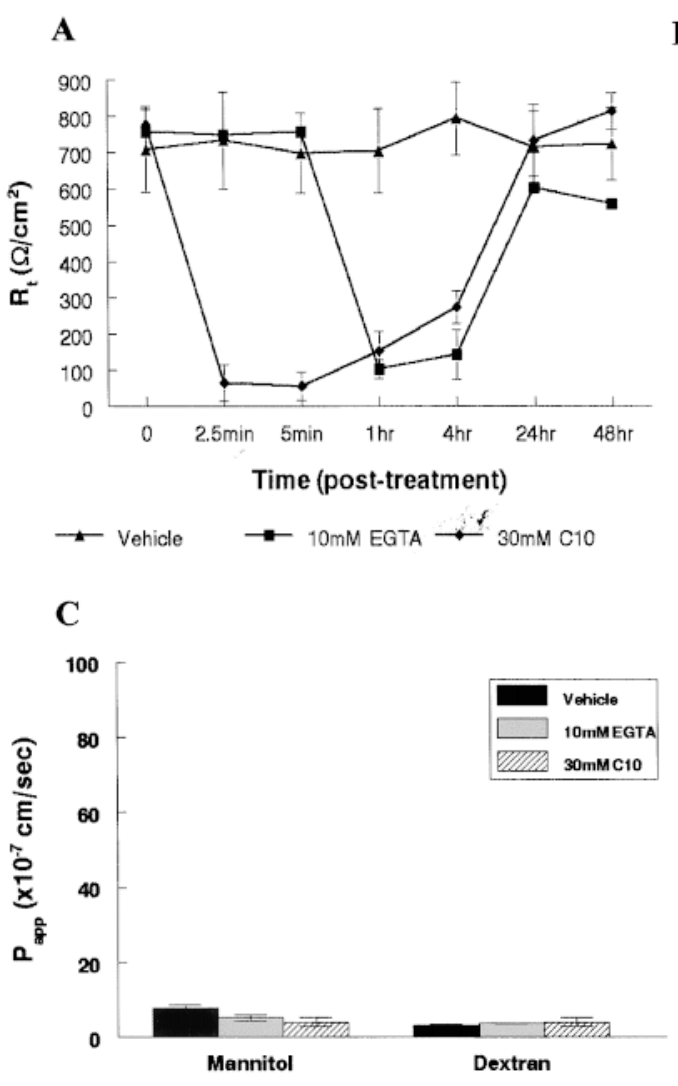

$\mathbf{B}$

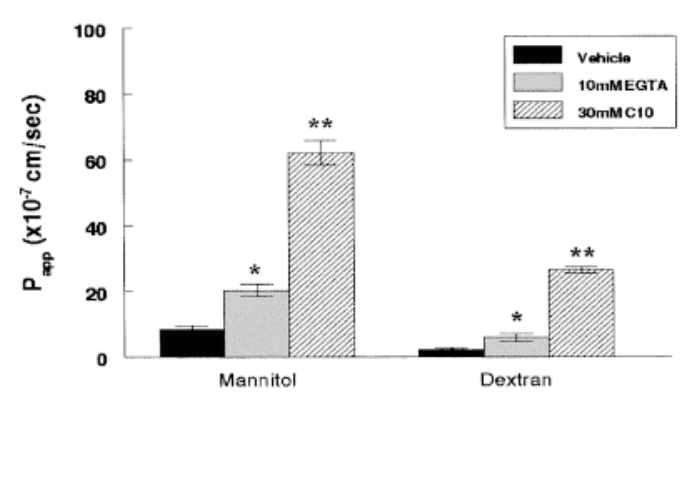

Figure 1. Effect of EGTA and $\mathrm{C} 10$ on $\mathrm{R}_{\mathrm{t}}$ and $\mathrm{P}_{\text {app. }}$ (A) $\mathrm{R}_{\mathrm{t}}$ of polarized primary HAE cells before and after treatment with vehicle, $10 \mathrm{mM}$ EGTA, or $30 \mathrm{mM} \mathrm{C10.} \mathrm{(B)} \mathrm{Apparent} \mathrm{P}_{\text {app }}$ coefficient of HAE cells directly after treatment with vehicle, $10 \mathrm{mM}$ EGTA, or $30 \mathrm{mM} \mathrm{C10.} \mathrm{(C)} \mathrm{P}_{\text {app }}$ of HAE cells $24 \mathrm{~h}$ after treatment. *Significantly different from vehicle and significantly different from both vehicle-* and EGTA-treated $* *$ cells at $P<0.001$. 

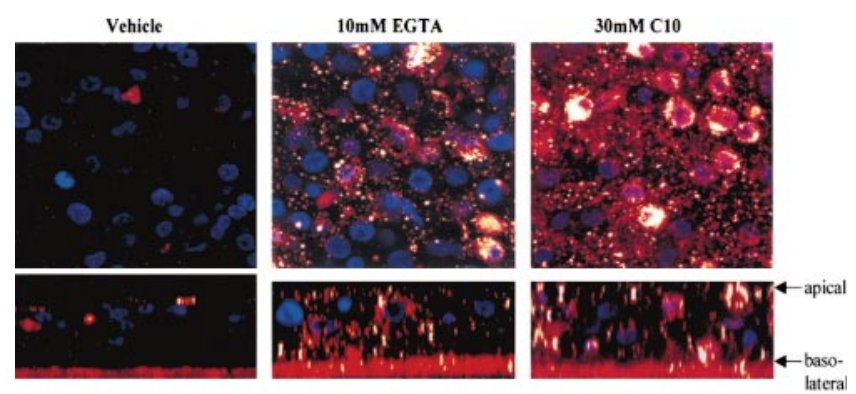

Figure 2. Localization of Cy3-AdlacZ. Primary HAE cells were incubated with Cy3-labeled AdlacZ on the apical surface after treatment with vehicle, $10 \mathrm{mM}$ EGTA, or $30 \mathrm{mM} \mathrm{C10}$. Cultures were washed with PBS, and labeled vector was localized by confocal microscopy. Blue color represents 4',6-diamidino-2-phenylindole-stained nuclei and red color represents $\mathrm{Cy} 3$-adenovirus particles. Top row: xy image; bottom row: xz image.

\section{Effect of EGTA and C10 on Vector Penetration}

To determine whether the increase in permeability following treatment with EGTA and C10 correlated with an increase in vector penetration, we measured the penetration of fluorescence $(\mathrm{Cy} 3)$ labeled $\mathrm{Ad} l a c Z$ vectors into cultures. Primary HAE cells were pretreated with EGTA, $\mathrm{C} 10$, or vehicle, and were then infected with Cy3-AdlacZ at an MOI of 100. The localization of Cy3-adenovirus particles in vehicle-treated cultures was restricted to the apical surfaces of the cells, with very little vector penetration to the basolateral surface (Figure 2). However, in cells pretreated with either EGTA or C10, viral particles were seen throughout the epithelium, with high levels penetrating to the basolateral surface (Figure 2). These results show that transient permeabilization of the intercellular junctional complex by EGTA or C10 permits vector particles to penetrate to the basolateral surface, where subsequent viral binding and internalization may occur.

\section{Enhanced AdlacZ- and AdCFTR-Mediated Gene Transfer}

Since modifying the junctional complex by treatment with EGTA or C10 increased the ability of Cy3-Ad vectors to penetrate to the basolateral surface, we investigated the effects of these agents on adenovirus-mediated gene transfer to the airway epithelium. Following treatment with vehicle, EGTA, or C10, $\mathrm{R}_{\mathrm{t}}$ was reduced (Figure $3 \mathrm{~A}$ ), and the apical surface was infected with Adlac $Z$ at an MOI of 100. Pretreatment of HAE cells with EGTA or C10 significantly enhanced the level of adenovirus-mediated $\beta$-galactosidase expression as compared with that of controls (Figure $3 \mathrm{~B})$. Cultures treated with vehicle alone generated $\beta$-galactosidase activity of $15 \pm 2$ (mean \pm SEM) $\mathrm{mU} \beta$-galactosidase/mg protein. In contrast, EGTA-treated cultures showed an activity of $120 \pm 10 \mathrm{mU} \beta$-galactosidase $/ \mathrm{mg}$ protein, and $\mathrm{C} 10$-treated cultures exhibited an activity of $480 \pm 105 \mathrm{mU} \beta$-galactosidase $/ \mathrm{mg}$ protein. Therefore, both EGTA and C10 caused a significant increase in adenovirus-mediated gene transfer to HAE cells, whereas vehicle alone did not. C10 pretreatment also led to signifi-
$\mathbf{A}$

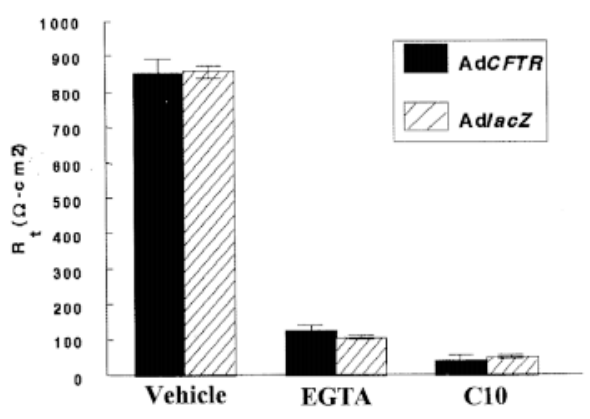

C

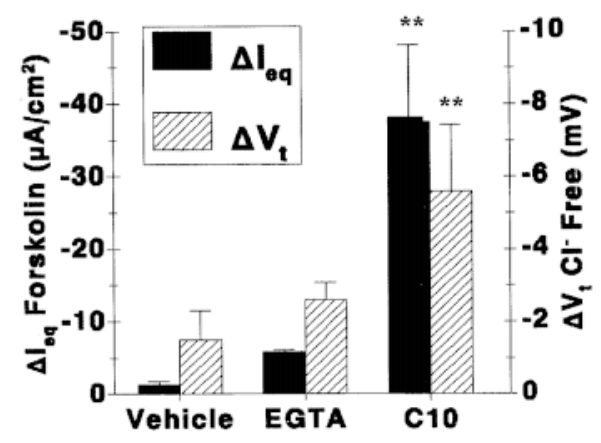

B

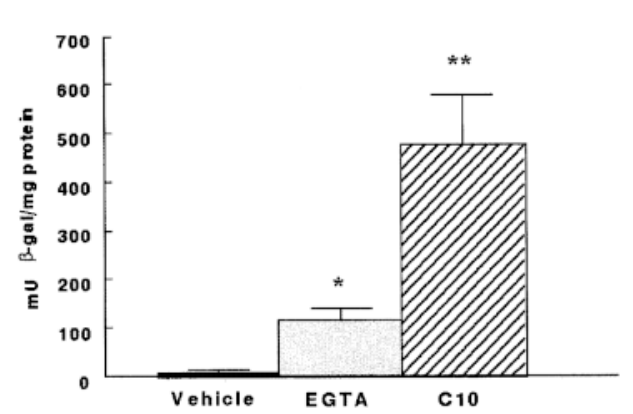

D

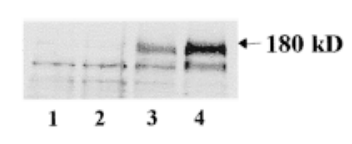

Figure 3. Transduction efficiency. $(A) \mathrm{R}_{\mathrm{t}}$ following treatment with vehicle, EGTA, or $\mathrm{C} 10$ and preceding infection with AdlacZ or AdCFTR. (B) Adlac $Z$ transduction. Primary HAE cells were treated with vehicle, $10 \mathrm{mM}$ EGTA, or 30 $\mathrm{mM} \mathrm{C10}$ and were infected with AdlacZ. Shown is transgene expression as determined by $\beta$-galactosidase enzyme assay. (C) CFTR-mediated $\mathrm{Cl}^{-}$secretory responses in vehicle-, EGTA-, and C10-treated cultures. Change in voltage in response to luminal $\mathrm{Cl}^{-}$substitution $\left(\Delta \mathrm{V}_{\mathrm{t}}, \mathrm{Cl}^{-}\right.$-free $)$and change in equivalent shortcircuit current in response to forskolin $\left(\Delta \mathrm{I}_{\mathrm{eq}}\right.$ forskolin) are shown. $(D)$ Western blot analysis of total protein from cultures in $C$. Protein was detected with a polyclonal rabbit antibody recognizing the $\mathrm{R}$ domain of CFTR. Lane 1: uninfected culture treated with vehicle; lane 2 : culture treated

with vehicle and infected with AdCFTR; lane 3: culture treated with $10 \mathrm{mM}$ EGTA and infected with AdCFTR; lane 4: cultures treated with $30 \mathrm{mM} \mathrm{C10}$ and infected with AdCFTR. Significantly different from vehicle-* and significantly different from both vehicle- and EGTA-treated $* *$ cells, at $P<0.001$. 
cantly higher levels of $\beta$-galactosidase activity than did pretreatment with EGTA.

To determine whether $\mathrm{C} 10$ could enhance the AdCFTR-mediated functional correction of CF epithelia, we infected vehicle-exposed (untreated), EGTA-treated, or C10-treated CF primary HAE cells with AdCFTR (MOI of 300 ) after $R_{t}$ was reduced (Figure $3 \mathrm{~A}$ ). The effects on basal and stimulated CFTR-mediated $\mathrm{Cl}^{-}$secretion at $48 \mathrm{~h}$ after AdCFTR transduction are presented in Figure 3C. C10treated cultures infected with AdCFTR exhibited a change in voltage in response to luminal $\mathrm{Cl}^{-}$substitution $\left(\Delta \mathrm{V}_{\mathrm{tCl}^{-} \text {-free }}\right)$ of $-28 \pm 1.7 \mathrm{mV}$, as compared with $-8 \pm 0.8 \mathrm{mV}$ in untreated CF HAE cell cultures and $-14 \pm 3 \mathrm{mV}$ in EGTAtreated cultures. Similarly, the change in equivalent shortcircuit current in response to forskolin $\left(\Delta \mathrm{I}_{\mathrm{eq}}\right.$ forskolin) in C10-treated cultures was $-38 \pm 17 \mu \mathrm{A} / \mathrm{cm}^{2}$, in comparison with $-3 \pm 0.8 \mu \mathrm{A} / \mathrm{cm}^{2}$ in vehicle treated-CF cultures, and was $-6 \pm 0.6 \mu \mathrm{A} / \mathrm{cm}^{2}$ in EGTA-treated cultures.

To confirm expression of the CFTR, we performed Western blot analysis on lysates generated from these same cultures after bioelectric characterization (Figure 3D). In vehicle-control CF cultures not infected with AdCFTR, there was an absence of CFTR (Figure 3D, lane 1). When cultures were infected with AdCFTR, there was no protein expression in tissues treated with vehicle alone (Figure 3D, lane 2), but high levels of expression were seen in both EGTA- and C10-treated cultures (Figure 3D, lanes 3 and 4 , respectively). In accord with the bioelectric results, CFTR protein expression in C10-treated cultures was greater than in cultures treated with EGTA.

\section{Modulation of Junctional Permeability and Cytokine Secretion}

Because high levels of inflammatory cytokines occur in $\mathrm{CF}$ secretions, we examined whether EGTA and C10 induced cytokine secretion in normal and CF primary HAE cells. Medium was collected from the apical and basolateral compartments of both normal and CF HAE cell cultures at $4 \mathrm{~h}$ after treatment with vehicle, EGTA, or C10. Treatment of normal or CF HAE cells with EGTA or C10 did not cause a significant increase in levels of IL-6 (Figure 4A) or IL-8 (Figure 4B) secretion, whereas a significant increase in IL-8
A

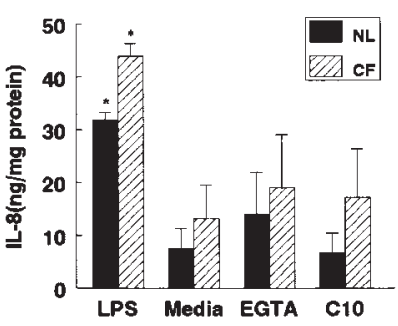

B

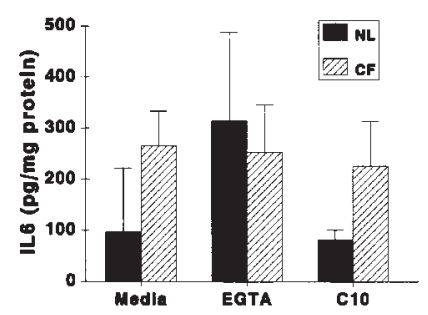

Figure 4. Cytokine response to EGTA and C10 treatment. Primary HAE cells treated with vehicle, $10 \mathrm{mM}$ EGTA, or $30 \mathrm{mM}$ C10. As a positive control, parallel cultures were treated with $5 \mu \mathrm{g}$ LPS for $1 \mathrm{~h}$. Shown is the amount of cytokine released into the medium at $4 \mathrm{~h}$ after treatment. $(A)$ IL-8 and $(B)$ IL-6. Data are presented as total amount of cytokine released (luminal + basolateral medium) in six cultures. Significantly different from vehicle-, EGTA-, and C10-treated cultures*, at $P<0.001$. secretion was detected in the positive controls treated with LPS.

\section{Effects of EGTA and C10 on the Integrity of TJ-Associated Proteins}

To determine whether EGTA and/or C10 disrupted TJassociated proteins, we performed immunofluorescence localization and Western blot analyses of ZO-1, occludin, E-cadherin, and claudin-1. In epithelium and endothelium, an intact $\mathrm{TJ}$ is characterized by a continuous ring of $\mathrm{TJ}-$ associated proteins that circumscribes each cell when examined by immunofluorescence microscopy.

When immunofluorescence localization was done for the cytoplasmic TJ protein ZO-1, we saw no apparent disruption of its typical staining pattern after exposure to EGTA or C10 (Figure 5A). There was an increase in the staining intensity after treatment with both EGTA $(167 \%$ over the control value) and C10 (143\% over the control value). To determine whether this increase in staining intensity could be correlated with an increase in protein expression, we performed Western blot analysis. As seen in Figure 5A, lane 2, vehicle-treated cultures showed expression of both the $\alpha^{+}$and $\alpha^{-}$isoforms of ZO-1. However, after treatment with $10 \mathrm{mM}$ EGTA, the $\alpha^{-}$isoform was no longer apparent (Figure 5A, lane 3). On the other hand, after treatment with $\mathrm{C} 10$, there was no change in isoform expression (Figure 5A, lane 4).

Occludin immunofluorescence in primary HAE cells also showed no apparent disruption after treatment with either EGTA or C10 (Figure 6A). However, the intensity of staining after treatment with either agent was enhanced (149\% relative to control levels following EGTA treatment, and $186 \%$ following C10 treatment). To further analyze this change in intensity, we tested the effect of EGTA or C10 on occludin expression through Western blot analysis (Figures 6B and 6C). Immediately after treatment with either $10 \mathrm{mM}$ EGTA (Figure 6B, lane 3) or $30 \mathrm{mM} \mathrm{C10}$ (Figure 6C, lane 3), the intensity of occludin staining detected by Western blotting was increased in comparison with that in vehicle-treated cultures (Figures 6B and 6C, lane 2). Over a period of $24 \mathrm{~h}$, these levels returned to control levels. To control for protein loading, we stripped these blots and reprobed with an antibody directed at E-cadherin (Figure 6D). In contrast to the case with either occludin or ZO-1, Western blot analysis of E-cadherin revealed no apparent changes in protein levels. Similarly, immunofluorescence microscopy for E-cadherin showed that it remained unchanged after treatment with either EGTA or C10 (data not shown).

The effects of EGTA or C10 on the expression and localization of claudin-1 in primary HAE cells have not been reported. To examine the effects of these agents on claudin-1 in HAE cells, we performed immunofluorescence microscopy and Western blot analysis of claudin-1. Claudin-1 localization was disrupted in HAE cells pretreated with C10, but not in cells pretreated with EGTA or vehicle (Figure 7A). No staining was detected in NIH 3T3 cells, which served as a negative control. Western blot analysis of claudin-1 revealed no apparent change in the expression of this protein after treatment with EGTA or C10 
A
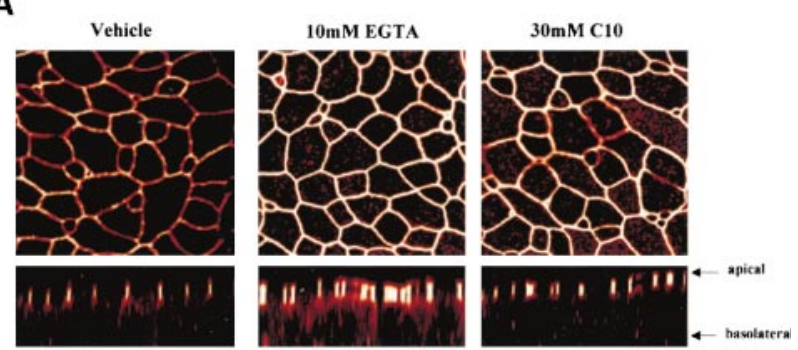

B

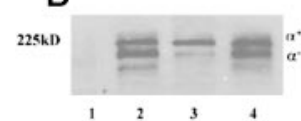

Figure 5. ZO-1 localization and expression. $(A)$ Immunofluorescence staining for ZO-1 as detected by confocal microscopy in vehicle-, EGTA-, or C10-treated primary HAE cells. Top row: xy image; bottom row: $\mathrm{xz}$ image. $(B)$ Primary HAE cells treated with vehicle (lane 2), $10 \mathrm{mM}$ EGTA (lane 3), or $30 \mathrm{mM} \mathrm{C10} \mathrm{(lane} \mathrm{4).}$ Protein was detected with an antibody to ZO-1. Lane 1: secondary antibody alone; lane 2: vehicle-treated cultures; lane 3: EGTA-treated cultures; and lane 4: C10-treated cultures.

(Figure 7B). Thus, although C10 and EGTA have similar effects on ZO-1 and occludin distribution, C10 clearly disrupts claudin-1 distribution, whereas EGTA does not.

\section{Discussion}

Previous studies have shown that well-differentiated airway epithelia are resistant to vector transduction from the apical surface. The airway epithelium prevents penetration of vector particles to the basolateral membrane, where uptake receptors are expressed. This barrier effectively limits the feasibility of gene therapy for the treatment of $\mathrm{CF}$. The findings presented here confirm that modulation of the TJ by divalent chelators can allow vector diffusion to the basolateral surface of airway epithelia, and can promote vector binding and entry.

In the search for more effective transduction-promoting agents than divalent chelators, we identified C10 as an agent that rapidly modulated the $\mathrm{R}_{\mathrm{t}}$ of the junctional complex and permitted full recovery (Figure 1A). The use of a medium-chain fatty acid to enhance gene transfer to airway epithelium has not been reported. EGTA and C10 decreased $R_{t}$ to similar levels, but their effects on the enhancement of gene transfer differed markedly. C10 caused a significantly greater increase in $\mathrm{P}_{\mathrm{app}}$ for both small and large nonpolar molecules, which lends evidence to the notion that this agent could be used to enhance gene transfer more efficiently than the previously identified EGTA.

Our comparison of the effects of EGTA and C10 on the transfer of both lacZ and CFTR cDNAs supported this concept. The effects of $\mathrm{C} 10$ on $\mathrm{Ad} l a c Z$ gene transfer to polarized airway epithelia were pronounced (Figure 3B). After treatment with $\mathrm{C} 10$, gene transfer efficiency increased by 50 -fold, as compared with an increase of 10 -fold following EGTA treatment. This greater increase in adenovirus-mediated gene transfer is further supported by the AdCFTR
A

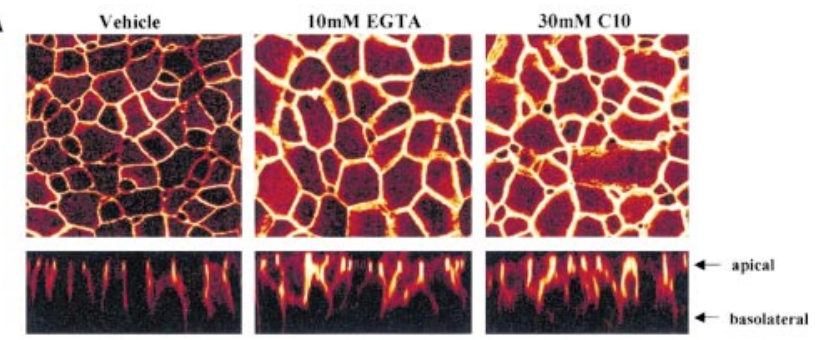

B

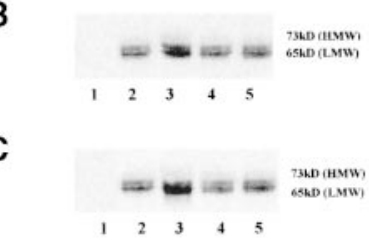

D

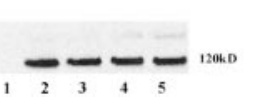

Figure 6. Occludin localization and expression. $(A) \mathrm{Im}$ munofluorescence staining for occludin as detected by confocal microscopy in vehicle-, EGTA-, or C10-treated primary HAE cells. Top: xy image; bottom: xz image. Western blot analysis of occludin after treatment with $10 \mathrm{mM}$ EGTA $(B)$ or $30 \mathrm{mM} \mathrm{C10}(C)$. Protein was detected with an antibody to occludin; lane 1: secondary alone; lane 2: immediately after vehicle treatment; lane 3: after EGTA or C10 treatment; lane 4: $12 \mathrm{~h}$ after EGTA or C10 treatment; lane 5: $24 \mathrm{~h}$ after EGTA or C10 treatment. HMW and LMW phosphorylation states of occludin. (D) To control for equal protein loading, membranes from $C$ were stripped and reprobed with a monoclonal antibody to E-cadherin.

transduction of polarized primary CF HAE cells observed in our study (Figures 4C and 4D). These findings provide evidence that C10 may be a better agent than EGTA for the enhancement of gene transfer in CF airways.

An obvious concern with the use of tight-junctionalregulating agents in vivo in $\mathrm{CF}$ is the potential inflammatory response elicited by exposure to these agents. To address this concern in vitro in cultures of both normal and

\section{A}
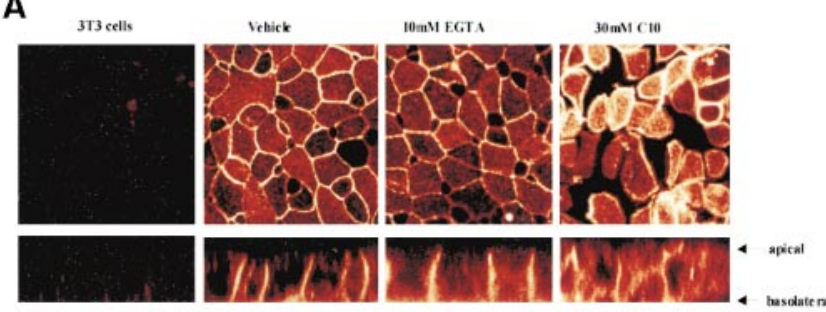

B

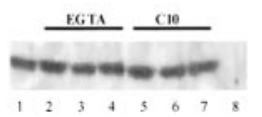

Figure 7. Claudin-1 localization and expression in primary HAE cells. $(A)$ Immunofluorescence staining for claudin-1 in primary HAE cells and NIH 3 T3 cells (negative control) as detected by confocal microscopy. Top: xy image; bottom: xz image. Note disruption after $\mathrm{C} 10$ treatment. $(B)$ Western blot analysis of claudin1 in primary HAE cells treated with EGTA or C10. Protein was detected with an antibody to claudin-1. Lane 1: immediately after vehicle treatment; lane 2: after EGTA treatment; lane 3: $12 \mathrm{~h}$ after EGTA treatment; lane 4: $24 \mathrm{~h}$ after $\mathrm{C} 10$ treatment; lane 5: after $\mathrm{C} 10$ treatment; lane 6: $12 \mathrm{~h}$ after $\mathrm{C} 10$ treatment; lane $7: 24 \mathrm{~h}$ after $\mathrm{C} 10$ treatment; and lane 8 : secondary antibody alone. 
CF HAE cells, we measured the amount of secretion of two inflammatory cytokines important in the pathogenesis of CF, IL-6 and IL-8. We found that there was no significant increase in the amount of cytokine secretion after treatment with EGTA or C10 (Figures 4A and 4B) over that in vehicle-treated cultures. In contrast, levels of IL-8 were increased after LPS treatment. These data indicate that EGTA and C10 do not themselves elicit significant inflammatory responses in either normal or CF primary HAE cells in vitro. However, we cannot, in this in vitro study, exclude the possibility that increasing epithelial permeability might allow access of luminal inflammatory components, including bacteria, into the interstitial compartment. C10 has known bactericidal properties that may potentially limit bacterially-induced inflammation, should this occur (14). However, this possibility can only be tested in an in vivo model (e.g., the Pseudomonas-induced model of bronchopulmonary inflammation [15]). Interestingly, luminal contents do not appear to induce an inflammatory response after rectal administration of ampicillin with $\mathrm{C} 10$ suppositories in humans (16).

Studies of the effects of $\mathrm{C} 10$ on proteins involved in modulating the permeability of the TJ have primarily focused on intestinal absorption, with Caco-2 cells serving as an epithelial model (16-18). Previous work with these cells suggests that the staining pattern of both ZO-1 and occludin is disrupted after exposure to C10 (16). To identify whether $\mathrm{C} 10$ acts on similar proteins in the airway epithelium, we performed immunofluorescence staining for ZO-1, occludin, E-cadherin, and claudin-1. In contrast to results reported with Caco- 2 cells, the results presented here suggest that the distribution of ZO-1 and occludin, as well as that of E-cadherin, is not affected in primary HAE cells after exposure to $\mathrm{C} 10$. Immunofluorescence staining for ZO-1 revealed no apparent effect of C10 or EGTA on ZO-1 organization, but an increase in the staining pattern was induced after treatment with both agents (Figure 5A). This increase in apparent staining intensity most likely reflects an increase in the localization of ZO-1 to the TJ. Western blot analysis, although its results did not correlate with an increase in ZO-1 expression, revealed a surprising effect of EGTA on the $\alpha^{-}$isoform of ZO-1 (Figure 5B). It has been shown that an alternative splicing pattern results in two isoforms of ZO-1 (ZO-1 $\alpha^{+}$and $\left.\alpha^{-}\right)$, which differ in an internal 80-residue domain (the $\alpha$ domain) $(19,20)$. Although there appears to be no correlation between the magnitude of $\mathrm{R}_{\mathrm{t}}$ and ZO-1 isoform expression, it has been speculated that cells expressing one or the other isoform of ZO-1 differ from one another in their degree of junctional plasticity. Published data suggest that expression of the $\alpha^{-}$isoform of ZO-1 may occur in TJs that are more dynamic (19). This effect on isoform expression, however, is not seen after treatment with $\mathrm{C} 10$, suggesting that $\mathrm{C} 10$ and EGTA work via distinct mechanisms.

Although occludin organization was also not disrupted, the intensity of fluorescence staining increased significantly in both C10- and EGTA-treated cultures, which correlated with an increase in the apparent expression level of occludin (Figure 6B). It seems improbable that this increase resulted from an increase in protein synthesis, particularly after treatment with $\mathrm{C} 10$, whose effects were detected within $2 \mathrm{~min}$ after treatment. It is more likely that the apparent increase in occludin detected by Western blot analysis corresponds with the phosphorylation state of the protein itself. Occludin exists in both a low-molecular-weight (LMW) and high-molecular-weight (HMW) form, with the HMW form corresponding to an increased state of phosphorylation and the formation of TJs (21). After treatment with either C10 or EGTA, there appears to be an increase in occludin expression corresponding to the reformation of the TJ. By 12 to $24 \mathrm{~h}$ after treatment, the junctions have resealed and the levels of the HMW and LMW forms of occludin have returned to control levels (Figure 6). Immunofluorescence localization of occludin after treatment with both EGTA and C10 indicated that there is an increase in occludin localization at the TJ, which might indicate resealing of the junction after treatment. The phosphorylation state of occludin has been suggested to play an important role in the reassembly of the TJ after its disruption $(22,23)$. Further studies are needed to investigate the potential importance of changes in phosphorylation after exposure to C10 and EGTA.

The claudin family has been suggested to comprise major structural components of the TJ. This family of multigene transmembrane proteins consists of at least 15 distinct members (24). Previous work suggests that allergens might penetrate the airway epithelium by means of a component that is capable of disrupting protein components of the TJ, and particularly claudin-1 (25). Although the investigators who conducted this work suggested that sites exist within claudin- 1 that may be cleaved after allergen exposure, they did not demonstrate actual disruption of claudin distribution by immunofluorescence or Western blot analysis. Little is known about the role of the claudin family in primary HAE cells. To address the potential role of the claudin multigene family in maintenance of the $\mathrm{TJ}$ in the airway, we tested the effect of $\mathrm{C} 10$ in comparison with that of EGTA on both the localization and expression of claudin-1. Although treatment of HAE cells with EGTA did not affect the staining pattern of claudin-1, treatment with $\mathrm{C} 10$ caused obvious disruptions in the staining pattern of claudin-1 in HAE cells (Figure 7A). Therefore, the disruption of claudin-1 correlated with disruption of the $\mathrm{TJ}$ as indexed by $\mathrm{R}_{\mathrm{t}}$ and $\mathrm{P}_{\mathrm{app}}$ measurements.

In summary, we examined whether a medium-chain fatty acid used to enhance drug delivery can enhance gene transfer in the airway. Although both EGTA and C10 caused a similar decrease in $\mathrm{R}_{\mathrm{t}}$ in HAE cells, C10 produced a significantly greater increase in adenovirus-mediated gene transfer. The capacity of this agent to facilitate an increase in the level of adenovirus-mediated gene transfer to the airway epithelium makes it an attractive candidate for in vivo approaches to diseases of the lung. Although the exact mechanism by which $\mathrm{C} 10$ does this has not been resolved, it appears to act by a distinct mechanism in the airway to disrupt the junctional complex. The discovery that claudin- 1 is disrupted after exposure of airway epithelial cells to $\mathrm{C} 10$ points to the importance of this newly discovered protein in maintaining junctional integrity. Future studies will clarify the possible mechanism of action of $\mathrm{C} 10$ in vivo and in vitro, and will assess the feasibility of its use for gene therapy of CF. 
Acknowledgments: The authors are grateful to Hong Ni for technical assistance. This work was supported by grants HL58342 and HL51818 from the National Heart, Lung, and Blood Institute, National Institutes of Health and a Cystic Fibrosis Foundation grant.

\section{References}

1. Duan, D., Y. Yue, Z. Yan, P. B. McCray, Jr., and J. F. Engelhardt. 1998. Polarity influences the efficiency of recombinant adenoassociated virus infection in differentiated airway epithelia. Hum. Gene. Ther. 9:2761-2776.

2. Zabner, J., A. J. Fasbender, T. Moninger, K. A. Poellinger, and M. J. Welsh. 1995. Cellular and molecular barriers to gene transfer by a cationic lipid. $J$. Biol. Chem. 270:18997-19007.

3. Wang, G., B. L. Davidson, P. Melchert, V. A. Slepushkin, H. H. van Es, M. Bodner, D. J. Jolly, and P. B. McCray, Jr. 1998. Influence of cell polarity on retrovirus-mediated gene transfer to differentiated human airway epithelia. J. Virol. 72:9818-9826.

4. Grubb, B. R., R. J. Pickles, H. Ye, J. R. Yankaskas, R. N. Vick, J. F. Engelhardt, J. M. Wilson, L. G. Johnson, and R. C. Boucher. 1994. Inefficient gene transfer by adenovirus vector to cystic fibrosis airway epithelia of mice and humans. Nature 371:802-806.

5. Walters, R. W., T. Grunst, J. M. Bergelson, R. W. Finberg, M. J. Welsh, and J. Zabner. 1999. Basolateral localization of fiber receptors limits adenovirus infection from the apical surface of airway epithelia. J. Biol. Chem. 274:10219-10226.

6. Pickles, R. J., D. McCarty, H. Matsui, P. J. Hart, S. H. Randell, and R. C. Boucher. 1998. Limited entry of adenovirus vectors into well-differentiated airway epithelium is responsible for inefficient gene transfer. J. Virol. 72:6014-6023.

7. Fuller, S., C. H. von Bonsdorff, and K. Simons. 1984. Vesicular stomatitis virus infects and matures only through the basolateral surface of the polarized epithelial cell line, MDCK. Cell 38:65-77.

8. Wang, G., V. Slepushkin, J. Zabner, S. Keshavjee, J. C. Johnston, S. L. Sauter, D. J. Jolly, T. W. Dubensky, Jr., B. L. Davidson, and P. B. McCray, Jr. 1999. Feline immunodeficiency virus vectors persistently transduce nondividing airway epithelia and correct the cystic fibrosis defect. J. Clin. Invest. 104:R55-R62.

9. Lindmark, T., Y. Kimura, and P. Artursson. 1998. Absorption enhancement through intracellular regulation of tight junction permeability by medium chain fatty acids in Caco-2 cells. J. Pharmacol. Exp. Ther. 284:362-369.

10. Yankaskas, J. R., C. U. Cotton, M. R. Knowles, J. T. Gatzy, and R. C. Boucher. 1985. Culture of human nasal epithelial cells on collagen matrix supports: a comparison of bioelectric properties of normal and cystic fibrosis epithelia. Am. Rev. Respir. Dis. 132:1281-1287.

11. Stutts, M. J., R. C. Boucher, P. A. Bromberg, and J. T. Gatzy. 1981. Effects of ammonium and nitrate salts on lon transport across the excised canine trachea. Toxicol. Appl. Pharmacol. 60:91-105.

12. Leopold, P. L., B. Ferris, I. Grinberg, S. Worgall, N. R. Hackett, and R. G. Crystal. 1998. Fluorescent virions: dynamic tracking of the pathway of adenoviral gene transfer vectors in living cells. Hum. Gene. Ther. 9:367-378.

13. Johnson, L. G., J. P. Mewshaw, H. Ni, T. Friedmann, R. C. Boucher, and J. C. Olsen. 1998. Effect of host modification and age on airway epithelial gene transfer mediated by a murine leukemia virus-derived vector. $J$. $V i$ rol. 72:8861-8872.

14. Kabara, J. J. 1978. Fatty acids and derivatives as antimicrobial agents-a review. In J. J. Kabara, editor. The pharmacological effect of lipids. American Oil Chemists' Society. 1-14.

15. Heeckeren, A., R. Walenga, M. W. Konstan, T. Bonfield, P. B. Davis. and T. Ferkol. 1997. Excessive inflammatory response of cystic fibrosis mice to bronchopulmonary infection with Pseudomonas aeruginosa. J. Clin. Invest. 100:2810-2815

16. Lindmark, T., J. D. Soderholm, G. Olaison, G. Alvan, G. Ocklind, and P. Artursson. 1997. Mechanism of absorption enhancement in humans after rectal administration of ampicillin in suppositories containing sodium caprate. Pharm. Res. 14:930-935.

17. Sakai, M., T. Imai, H. Ohtake, H. Azuma, M. Otagiri. 1997. Effects of absorption enhancers on the transport of model compounds in Caco-2 cell monolayers: assessment by confocal laser scanning microscopy. J. Pharmacol. Sci. 86:779-785.

18. Tomita, M. M. Hayashi, and S. Awazu. 1996. Absorption-enhancing mechanism of EDTA, caprate, and decanoylcarnitine in Caco-2 cells. J. Pharmacol. Sci. 85:608-611.

19. Balda, M. S., and J. M. Anderson. 1993. Two classes of tight junctions are revealed by ZO-1 isoforms. Am. J. Physiol. 264:C918-C924.

20. Willott, E., M. S. Balda, M. Heintzelman, B. Jameson, and J. M. Anderson. 1992. Localization and differential expression of two isoforms of the tight junction protein ZO-1. Am. J. Physiol. 262:C1119-C1124.

21. Wong, V. 1997. Phosphorylation of occludin correlates with occludin localization and function at the tight junction. Am. J. Physiol. 273:C1859C1867.

22. Tsukamoto, T., and S. K. Nigam. 1999. Role of tyrosine phosphorylation in the reassembly of occludin and other tight junction proteins. Am. J. Physiol. 276:F737-F750.

23. Farshori, P., and B. Kachar. 1999. Redistribution and phosphorylation of occludin during opening and resealing of tight junctions in cultured epithelial cells. J. Membr. Biol. 170:147-156.

24. Tsukita, S., and M. Furuse. 1999. Occludin and claudins in tight-junction strands: leading or supporting players? Trends Cell Biol. 9:268-273.

25. Wan, H., H. L. Winton, C. Soeller, E. R. Tovey, D. C. Gruenert, P. J. Thompson, G. A. Stewart, G. W. Taylor, D. R. Garrod, M. B. Cannell, and C. Robinson. 1999. Der p 1 facilitates transepithelial allergen delivery by disruption of tight junctions. J. Clin. Invest. 104:123-133. 